\title{
Analyzing probabilistic optimal power flow problem by cubature rules
}

\author{
Qing Xiao* \\ School of Information and Electrical Engineering, Hunan University of Science and Technology, \\ Xiangtan, Hunan Province, China
}

$\begin{array}{llll}\text { Received: } 18.08 .2021 & \text { Accepted/Published Online: } 01.12 .2021 & \text { Pinal Version: } 21.03 .2022\end{array}$

\begin{abstract}
This paper is devoted to revealing some properties of the probabilistic optimal power flow (POPF) problem. In conjunction with Hermite polynomial model, Nataf transformation is introduced to map POPF problem to the independent standard normal space. Firstly, a multivariate polynomial model is employed to represent the function relationship between POPF inputs and outputs. Then, moment matching equations are derived to characterize the uncertainty effects of POPF inputs on outputs; three cubature rules are derived to calculate statistical moments of POPF outputs. Finally, along with Monte Carlo simulation method, the proposed methods are tested on IEEE 57-bus system and IEEE 118-bus system, whereby it reveals some characteristics of the function relation between POPF inputs and outputs.
\end{abstract}

Key words: Probabilistic optimal power flow, Hermite polynomial model, moment matching equations, cubature rules

\begin{tabular}{|clll|}
\hline Nomenclature & & & \\
\hline POPF & probabilistic optimal power flow & QMCS & Quasi-Monte Carlo simulation \\
OPF & optimal power flow & LHS & Latin hypercube sampling \\
CDF & cumulative distribution function & UT & unscented transformation \\
MCS & Monte Carlo simulation & CR & cubature rule \\
PEM & point estimate method & & \\
\hline
\end{tabular}

\section{Introduction}

Due to the inherent uncertainty in power systems, the probabilistic optimal power flow (POPF) technique has been suggested to analyze the operation and planning of power systems [1,2]. POPF is developed by introducing the probability theory to the deterministic optimal power flow (OPF) calculation, and sets out to obtain the statistical information of OPF solutions. For the purpose of convenient expression, this paper treats random variables in the OPF model as POPF inputs, and regards OPF solutions as POPF outputs. Because each POPF output is implicitly related to input by the OPF model, the statistical information of POPF outputs should be calculated numerically.

According to the joint cumulative distribution function (CDF) of POPF inputs, Monte Carlo simulation (MCS) draws samples to simulate various possible scenarios of power system operating states, then it performs deterministic OPF calculations for each scenario to generate samples of POPF outputs [3, 4]. As long as enough

${ }^{*}$ Correspondence: qxiao@mail.hnust.edu.cn 
samples are generated, MCS can yield accurate estimates for statistical moments and distribution functions of POPF outputs. However, the high accuracy of MCS comes at the cost of a high computational burden. In order to improve the efficiency, one strategy is to employ the Karush-Kuhn-Tucker condition to group samples of MCS, such that the computational time can be saved [5]. Another approach is to use Sobol sequence[6, 7] or Latin hypercube sampling (LHS) [8] to generate samples of POPF inputs. In [9, 10], Markov chain Monte Carlo method is applied to draw samples from Sobol sequence for POPF computation, which demonstrates a higher efficiency than MCS. In [8], a rank correlation based LHS method is suggested for POPF computation, which requires a smaller sample size than MCS.

Note that Monte Carlo-type methods are generic algorithms, they are not POPF-oriented. If we can exploit some characteristics of POPF problem, it helps to develop more efficient and accurate algorithms for POPF computation. The approximation method offers a new approach to attack the POPF problem, its underlying idea is to employ a polynomial model to approximate the function relationship between POPF inputs and outputs. The framework of approximation method allows to develop a POPF-oriented polynomial model for POPF computation, the widely used surrogate method[11, 12] and point estimate method (PEM) $[13,14]$ are actually two different forms of the approximation method (In Appendix A, an example is presented to show the equivalence between these two methods). The surrogate method focuses on determining coefficients of the polynomial model, such that an explicit function can be established to relate POPF inputs and outputs; the PEM is devoted to choosing quadrature weights and points to match statistical moments of monomials in the polynomial model, whereby it can fully consider the uncertainty impact of POPF inputs on outputs; the main disadvantage of Zhao's PEM is that its accuracy cannot be further improved by adding quadrature points $[15]$.

The cumulant method and unscented transformation (UT) method may also be classified as the approximation method. The cumulant method performs one deterministic OPF calculation at mean values of POPF inputs, and establishes an explicit linear model to relate POPF inputs and outputs [16]; this algorithm is very efficient and behaves well when the stochastic variation of POPF inputs is not large. The UT method also selects quadrature weights and points by using statistical moments of POPF inputs[17], which shows some similarity with PEM. When tackling POPF problem with correlated inputs, UT method considers the POPF problem in the original probability space, while PEM often employs copula method to map POPF problem to an independent and identically distributed probability space. If Gauss-type quadrature rule is introduced to determine the accuracy controlling parameters of UT, these two algorithms are identical[18].

Following the framework of the approximation method, this paper is devoted to revealing some properties of POPF problem. Compared with the existing works, the contribution of this paper is threefold:

1) It derives three new cubature rules to calculate statistical moments of POPF outputs.

2) By analyzing the performance of MCS and cubature rules for POPF computation, it identifies some characteristics of the function relationship between POPF inputs and outputs.

3) In the context of moment matching equations, it gives some suggestions for deriving new algorithms for POPF computation.

In order to clearly illustrate aforementioned algorithms and the proposed methods, their advantages and disadvantages are summarized in Table 1. 
Table 1. A summary of different algorithms for POPF computation.

\begin{tabular}{|c|c|c|c|}
\hline & Points & Advantages & Disadvantages \\
\hline MCS & $\begin{array}{l}\text { pseudo-random } \\
\text { sequence }\end{array}$ & $\begin{array}{l}\text { the accuracy can be improved by using } \\
\text { more points, and an error bound can be } \\
\text { given. }\end{array}$ & $\begin{array}{l}\text { the convergence rate is slow, a large } \\
\text { sample size is required to yield accurate } \\
\text { results. }\end{array}$ \\
\hline QMCS & Sobol sequence & \multirow{2}{*}{$\begin{array}{l}\text { it has a faster convergence rate than } \\
\text { MCS. }\end{array}$} & \multirow{2}{*}{$\begin{array}{l}\text { a theoretical error bound cannot be given, } \\
\text { and the sample size is not small for POPF } \\
\text { problem with a large number of inputs. }\end{array}$} \\
\hline LHS & $\begin{array}{l}\text { low discrepancy } \\
\text { sequence }\end{array}$ & & \\
\hline $\begin{array}{l}\text { cumulant } \\
\text { method }\end{array}$ & - & $\begin{array}{l}\text { it employs a linear model to relate POPF } \\
\text { inputs and outputs, and has a very high } \\
\text { efficiency. }\end{array}$ & $\begin{array}{l}\text { it does not work well for POPF problem } \\
\text { with highly variable inputs, and the } \\
\text { accuracy cannot be improved. }\end{array}$ \\
\hline $\begin{array}{l}\mathrm{UT} \\
\text { method }\end{array}$ & $\begin{array}{l}\text { moments of } \\
\text { POPF inputs }\end{array}$ & \multirow{2}{*}{$\begin{array}{l}\text { it behaves better than cumulant method, } \\
\text { and the computational burden increases } \\
\text { linearly with the number of POPF inputs. }\end{array}$} & \multirow[t]{2}{*}{$\begin{array}{l}\text { the accuracy cannot be further } \\
\text { improved. }\end{array}$} \\
\hline PEM & $\begin{array}{l}\text { Gauss-type } \\
\text { quadrature rule }\end{array}$ & & \\
\hline $\begin{array}{l}\text { Proposed } \\
\text { method }\end{array}$ & cubature rule & $\begin{array}{l}\text { it is more accurate than PEM, the } \\
\text { computational burden increases } \\
\text { linearly with the number of POPF inputs. }\end{array}$ & $\begin{array}{l}\text { it requires to construct an efficient and } \\
\text { accurate cubature rule. }\end{array}$ \\
\hline
\end{tabular}

\section{Problem formulation}

\subsection{Hermite polynomial model based Nataf transformation}

In this section, a Hermite polynomial model based Nataf transformation is presented to simulate different stochastic environments of POPF problem.

Let $X$ denote a random variable in the optimal power flow (OPF) model, the stochastic behavior of $X$ can be characterized by the following Hermite polynomial model [19]:

$$
X=\Omega(Z)=\mu+\frac{\sigma\left[H_{1}(Z)+a_{2} H_{2}(Z)+a_{3} H_{3}(Z)\right]}{\sqrt{6 a_{3}^{2}+2 a_{2}^{2}+1}}
$$

where $\mu$ and $\sigma$ are the mean and standard deviation of $X$ respectively, $Z$ is a standard normal variable, $\Omega(\cdot)$ is the function relationship between $X$ and $Z$. $H_{k}(Z)(k=1,2,3)$ are $k$ th-order Hermite polynomials:

$$
H_{1}(Z)=Z, \quad H_{2}(Z)=Z^{2}-1, \quad H_{3}(Z)=Z^{3}-3 Z
$$

$a_{2}, a_{3}$ are coefficients determined by the skewness $\gamma_{3}$ and kurtosis $\gamma_{4}$ of $X[19]$ :

$$
\begin{aligned}
a_{2}= & 0.1967 \gamma_{3}-0.01646 \gamma_{3} \gamma_{4}+0.01809 \gamma_{3}^{3}+7.438 \times 10^{-4} \gamma_{3} \gamma_{4}^{2}-9.209 \times 10^{-4} \gamma_{3}^{3} \gamma_{4}-1.366 \times 10^{-5} \gamma_{3} \gamma_{4}^{3}+ \\
& 1.527 \times 10^{-4} \gamma_{3}^{5}+1.07 \times 10^{-5} \gamma_{3}^{3} \gamma_{4}^{2}+8.823 \times 10^{-8} \gamma_{3} \gamma_{4}^{4} \\
a_{3}= & 0.0721+0.03176 \gamma_{4}-0.02942 \gamma_{3}^{2}-0.00179 \gamma_{4}^{2}+0.002348 \gamma_{3}^{2} \gamma_{4}+5.965 \times 10^{-5} \gamma_{4}^{3}-6.282 \times 10^{-4} \gamma_{3}^{4}- \\
& 6.355 \times 10^{-5} \gamma_{3}^{2} \gamma_{4}^{2}-9.692 \times 10^{-7} \gamma_{4}^{4}-0.07210 \gamma_{3}^{4} \gamma_{4}-0.07211 \gamma_{3}^{2} \gamma_{4}^{3}-0.07212 \gamma_{4}^{5}
\end{aligned}
$$

Let $X_{i}$ and $X_{j}$ be two correlated random variables in the OPF model, whose linear correlation coefficient is $\rho_{x}$. According to Eq. (1), $X_{i}$ and $X_{j}$ can be simulated by two correlated standard normal variables $Z_{i}$ and 
$Z_{j}$ respectively:

$$
\begin{aligned}
& X_{i}=\Omega_{i}\left(Z_{i}\right)=\mu_{i}+\frac{\sigma_{i}\left[H_{1}\left(Z_{i}\right)+a_{i, 2} H_{2}\left(Z_{i}\right)+a_{i, 3} H_{3}\left(Z_{i}\right)\right]}{\sqrt{6 a_{i, 3}^{2}+2 a_{i, 2}^{2}+1}}, \\
& X_{j}=\Omega_{j}\left(Z_{j}\right)=\mu_{j}+\frac{\sigma_{j}\left[H_{1}\left(Z_{j}\right)+a_{j, 2} H_{2}\left(Z_{j}\right)+a_{j, 3} H_{3}\left(Z_{j}\right)\right]}{\sqrt{6 a_{j, 3}^{2}+2 a_{j, 2}^{2}+1}} .
\end{aligned}
$$

Let $\rho_{z}$ be the correlation coefficient between $Z_{i}$ and $Z_{j}$. By using the orthogonal property of Hermite polynomial, the following formula can be derived to determine $\rho_{z}$ for a given $\rho_{x}[20]$ :

$$
\rho_{x}=\frac{a_{i, 3} a_{j, 3} \rho_{z}^{3}+a_{i, 2} a_{j, 2} \rho_{z}^{2}+\rho_{z}}{\sqrt{\left(6 a_{i, 3}^{2}+2 a_{i, 2}^{2}+1\right)\left(6 a_{j, 3}^{2}+2 a_{j, 2}^{2}+1\right)}}, \quad\left(\rho_{x} \rho_{z} \geq 0, \quad\left|\rho_{z}\right| \leq 1\right) .
$$

Denote all random variables in the OPF model as an $m$-dimensional random vector $\boldsymbol{X}=\left(X_{1}, \ldots, X_{i}, \ldots, X_{m}\right)$, $\boldsymbol{X}$ can be related to an independent standard normal vector $\boldsymbol{U}=\left(U_{1}, \ldots, U_{i}, \ldots, U_{m}\right)$ by following procedures:

1) For $\rho_{x}(i, j)$ between $X_{i}$ and $X_{j}$ in $\boldsymbol{X}(i, j=1, \ldots, m)$, determine the corresponding $\rho_{z}(i, j)$ by Eq.(2), construct a correlation matrix $\boldsymbol{R}_{\boldsymbol{Z}}=\left\{\rho_{z}(i, j)\right\}$, perform Cholesky decomposition on $\boldsymbol{R}_{\boldsymbol{Z}}$ to obtain the lower triangular matrix $\boldsymbol{L}$ :

$$
R_{Z}=L L^{T}
$$

2) The correlated standard normal vector $\boldsymbol{Z}=\left(Z_{1}, \ldots, Z_{i}, \ldots Z_{m}\right)$ can be related to an independent standard normal vector by:

$$
\boldsymbol{Z}=\boldsymbol{L} \boldsymbol{U}
$$

3) Transform $\boldsymbol{Z}$ to $\boldsymbol{X}$ by Eq.(1).

The above algorithm is also known as Nataf transformation, which can be expressed as [21]:

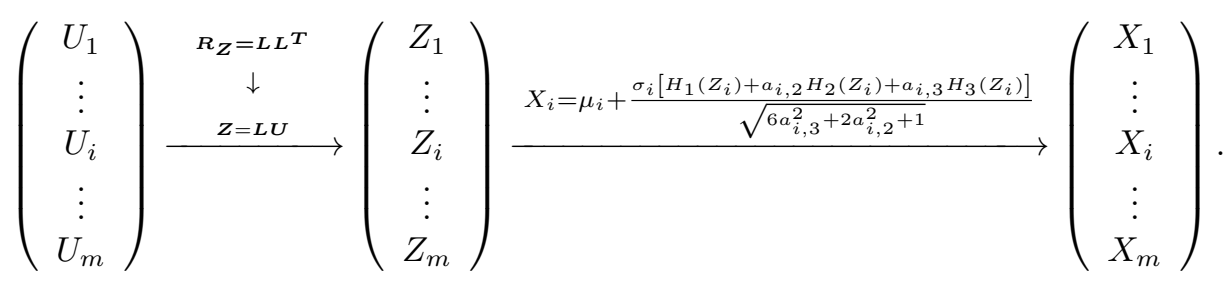




\subsection{POPF computation}

Consider an OPF model aiming to maximize social welfare:

$$
\begin{aligned}
& \text { Min: } \quad-\left(\boldsymbol{C}_{\boldsymbol{D}}^{\boldsymbol{T}} \boldsymbol{P}_{\boldsymbol{D}}-\boldsymbol{C}_{\boldsymbol{S}}^{\boldsymbol{T}} \boldsymbol{P}_{\boldsymbol{S}}\right) \quad \rightarrow \text { social welfare } \\
& \text { s.t. } \boldsymbol{g}\left(\boldsymbol{\theta}, \boldsymbol{V}, \boldsymbol{Q}_{\boldsymbol{G}}, \boldsymbol{P}_{\boldsymbol{S}}, \boldsymbol{P}_{\boldsymbol{D}}\right)=\mathbf{0} \rightarrow \text { power flow Eqs. } \\
& \boldsymbol{P}_{\boldsymbol{D m i n}} \leq \boldsymbol{P}_{\boldsymbol{D}} \leq \boldsymbol{P}_{\boldsymbol{D m a x}} \rightarrow \text { demand bid limits } \\
& \boldsymbol{P}_{\boldsymbol{S m i n}} \leq \boldsymbol{P}_{\boldsymbol{S}} \leq \boldsymbol{P}_{\boldsymbol{S m a x}} \rightarrow \text { supply bid limits } \\
& \boldsymbol{Q}_{\boldsymbol{G m i n}} \leq \boldsymbol{Q}_{\boldsymbol{G}} \leq \boldsymbol{Q}_{\boldsymbol{G m a x}} \rightarrow \text { generator limits } \\
& \left|P_{i j}(\theta, V)\right| \leq P_{i j \max } \rightarrow \text { "security"limits } \\
& \left|P_{j i}(\theta, V)\right| \leq P_{j i \max } \\
& \left|I_{i j}(\theta, V)\right| \leq I_{i j \max } \quad \rightarrow \text { thermal limits } \\
& \left|I_{j i}(\theta, V)\right| \leq I_{j i \max } \\
& \boldsymbol{V}_{\min } \leq \boldsymbol{V} \leq \boldsymbol{V}_{\max } \quad \rightarrow \text { voltage limits }
\end{aligned}
$$

where $\boldsymbol{C}_{\boldsymbol{D}}$ and $\boldsymbol{C}_{\boldsymbol{S}}$ are vectors of supply and demand bids in $\$ /$ MWh respectively, $\boldsymbol{P}_{\boldsymbol{D}}$ and $\boldsymbol{P}_{\boldsymbol{S}}$ are bounded supply and demand power bids in MW, $\boldsymbol{V}$ and $\boldsymbol{\theta}$ represent the bus phasor voltages and angles respectively, $\boldsymbol{Q}_{\boldsymbol{G}}$ is the generator reactive powers; $P_{i j}\left(P_{j i}\right)$ represents the power flowing from bus $i(j)$ to bus $j(i)$, and are used to indicate system security by limiting the transmission line power flows, together with line current $I_{i j}$ and $I_{j i}$ thermal limits and bus voltage limits.

If some variables in the OPF model are random variables, solutions of the OPF model would also be random variables. Denote an arbitrary solution of OPF model as $Y$, it has[8]:

$$
Y=H(\boldsymbol{X})=H\left(X_{1}, \ldots, X_{i}, \ldots, X_{m}\right)
$$

where $X_{i}(i=1, \ldots, m)$ are random variables in the OPF model, which are referred to as POPF inputs; $Y$ is referred to as POPF outputs. By using Nataf transformation in Eq.(5), $Y$ can be expressed as a function of independent standard normal variables:

$$
Y=H\left[\Omega_{1}\left(X_{1}\right), \ldots, \Omega_{i}\left(X_{i}\right), \ldots, \Omega_{m}\left(X_{m}\right)\right]=G\left(U_{1}, \ldots, U_{i}, \ldots, U_{m}\right)
$$

Then, the $k$ th-order raw moment of $Y$ is calculated by

$$
E\left[Y^{k}\right] \simeq \sum_{s=1}^{n} p_{s} G^{k}\left(\boldsymbol{t}_{s}\right)=\sum_{s=1}^{n} p_{s} G^{k}\left(t_{s, 1}, \ldots, t_{s, i}, \ldots, t_{s, m}\right)
$$

where $\boldsymbol{t}_{s}=\left(t_{s, 1}, \ldots, t_{s, i}, \ldots, t_{s, m}\right)$ is the $s$ th quadrature point, $p_{s}$ is the weight of $\boldsymbol{t}_{s}$. If $G^{k}(\cdot)$ in Eq.(9) is represented by a multivariate polynomial, $p_{s}$ and $\boldsymbol{t}_{s}$ should meet the following equations [22]:

$$
\sum_{s=1}^{n} p_{s} t_{s, 1}^{l_{1}} \cdots t_{s, i}^{l_{i}} \cdots t_{s, m}^{l_{m}}=E\left[U_{1}^{l_{1}} \cdots U_{i}^{l_{i}} \cdots U_{m}^{l_{m}}\right]=\prod_{i=1}^{m} E\left[U_{i}^{l_{i}}\right] .
$$


Denote $l_{i}=2 r_{i}-1$, or $2 r_{i}$, the moment matching equations in Eq.(10) can be classified into four types:

$$
\begin{aligned}
& \sum_{s=1}^{n} p_{s} t_{s, i}^{2 r_{i}-1}=0 \\
& \sum_{s=1}^{n} p_{s} t_{s, i}^{2 r_{i}}=E\left[U_{i}^{2 r_{i}}\right] \\
& \sum_{s=1}^{n} p_{s} t_{s, 1}^{l_{1}} \cdots t_{s, i}^{2 r_{i}-1} \cdots t_{s, m}^{l_{m}}=0 \\
& \sum_{s=1}^{n} p_{s} t_{s, 1}^{2 r_{1}} \cdots t_{s, i}^{2 r_{i}} \cdots t_{s, m}^{2 r_{m}}=\prod_{i=1}^{m} E\left[U_{i}^{2 r_{i}}\right] .
\end{aligned}
$$

If $p_{s}$ and $\boldsymbol{t}_{s}$ can satisfy more equations in Eqs.(11)-(14), the quadrature rule is expected to yield more accurate estimates for $E\left[Y^{k}\right]$ in Eq.(9).

\section{Cubature rules}

In this section, it develops three quadrature rules to calculate $E\left[Y^{k}\right]$ in Eq.(9), which are denoted as CR-I, CR-II, and CR-III respectively.

\subsection{Cubature rule of Type-I}

This part aims to develop a quadrature rule with an algebraic accuracy of degree 5 , the weights and points are shown in Table 2.

Table 2. The quadrature weights and points of cubature rule of Type-I.

\begin{tabular}{|l|l|l|l|l|l|l|}
\hline \multirow{2}{*}{ Number } & Weight & \multicolumn{3}{|l|}{ Point } \\
\cline { 2 - 7 } & $p_{s}$ & $t_{s, 1}$ & $t_{s, 2}$ & $t_{s, 3}$ & $\cdots$ & $t_{s, m}$ \\
\hline 1 & $W_{0}$ & 0 & 0 & 0 & $\cdots$ & 0 \\
\hline $2 m$ & $W_{1}$ & $\pm t_{1}$ & 0 & 0 & $\cdots$ & 0 \\
\hline$\left(\begin{array}{c}m \\
2\end{array}\right)$ & $W_{2}$ & $\pm t_{2}$ & $\pm t_{2}$ & 0 & $\cdots$ & 0 \\
\hline
\end{tabular}

The symmetric structure of CR-I ensures that all equations in Eq.(11) and Eq.(13) can be satisfied. By matching weights and points in Table 2 to equations in Eq.(12) and Eq.(14), it has:

$$
\begin{array}{r}
W_{0}+W_{1} \cdot 2 m+W_{2} \cdot\left(\begin{array}{c}
m \\
2
\end{array}\right)=E\left[U^{0}\right]=1 \\
2 W_{1} \cdot t_{1}^{2}+4 W_{2}(m-1) t_{2}^{2}=E\left[U^{2}\right]=1 \\
2 W_{1} \cdot t_{1}^{4}+4 W_{2}(m-1) t_{2}^{4}=E\left[U^{4}\right]=3 \\
4 W_{2} t_{2}^{4}=E\left[U_{i}^{2} U_{j}^{2}\right]=1
\end{array}
$$

Solving above equations gives

$$
W_{0}=\frac{2}{m+2}, \quad W_{1}=\frac{4-m}{2(m+2)^{2}}, \quad t_{1}=\sqrt{m+2}, \quad W_{2}=\frac{1}{(m+2)^{2}}, \quad t_{2}=\sqrt{\frac{m+2}{2}} .
$$


The number of points of CR-I is

$$
n=2 m^{2}+1
$$

\subsection{Cubature rule of Type-II}

Denote

$$
\boldsymbol{T}_{1}=\left(\begin{array}{c}
t_{1} \\
-t_{1} \\
-t_{1} \\
t_{1}
\end{array}\right), \quad \boldsymbol{T}_{2}=\left(\begin{array}{c}
t_{2} \\
t_{2} \\
-t_{2} \\
-t_{2}
\end{array}\right), \quad \boldsymbol{O}=\left(\begin{array}{c}
0 \\
0 \\
0 \\
0
\end{array}\right), \quad \boldsymbol{w}=\left(\begin{array}{c}
w \\
w \\
w \\
w
\end{array}\right)
$$

Table 3. The quadrature weights and points of cubature rule of Type-II.

\begin{tabular}{|l|l|l|l|l|l|l|l|}
\hline \multirow{2}{*}{ Number } & Weight & \multicolumn{6}{|l|}{ Point } \\
\cline { 2 - 8 } & $p_{s}$ & $t_{s, 1}$ & $t_{s, 2}$ & $t_{s, 3}$ & $\cdots$ & $t_{s, m-1}$ & $t_{s, m}$ \\
\hline 1 & $1-4(m+1) w$ & 0 & 0 & 0 & $\cdots$ & 0 & 0 \\
\hline 4 & $\boldsymbol{w}$ & $\boldsymbol{T}_{1}$ & $\boldsymbol{O}$ & $\boldsymbol{O}$ & $\cdots$ & $\boldsymbol{O}$ & $\boldsymbol{O}$ \\
\hline 4 & $\boldsymbol{w}$ & $\boldsymbol{T}_{2}$ & $\boldsymbol{T}_{1}$ & $\boldsymbol{O}$ & $\cdots$ & $\boldsymbol{O}$ & $\boldsymbol{O}$ \\
\hline 4 & $\boldsymbol{w}$ & $\boldsymbol{O}$ & $\boldsymbol{T}_{2}$ & $\boldsymbol{T}_{1}$ & $\cdots$ & $\boldsymbol{O}$ & $\boldsymbol{O}$ \\
\hline & $\vdots$ & & & & & & \\
\hline 4 & $\boldsymbol{w}$ & $\boldsymbol{O}$ & $\boldsymbol{O}$ & $\boldsymbol{O}$ & $\cdots$ & $\boldsymbol{T}_{2}$ & $\boldsymbol{T}_{1}$ \\
\hline 4 & $\boldsymbol{w}$ & $\boldsymbol{O}$ & $\boldsymbol{O}$ & $\boldsymbol{O}$ & $\cdots$ & $\boldsymbol{O}$ & $\boldsymbol{T}_{2}$ \\
\hline
\end{tabular}

The cubature rule in Table 3 can commit all equations in Eq.(11) and Eq.(13). By matching weights and points in Table 3 to equations in Eq.(12) and Eq.(14), it has:

$$
\begin{gathered}
4 w\left(t_{1}^{2}+t_{2}^{2}\right)=E\left[U^{2}\right]=1 \\
4 w\left(t_{1}^{4}+t_{2}^{4}\right)=E\left[U^{4}\right]=3 \\
4 w t_{1}^{2} t_{2}^{2}=E\left[U_{i}^{2} U_{j}^{2}\right]=1
\end{gathered}
$$

Solutions of Eq.(18) are:

$$
w=\frac{1}{10}, \quad t_{1}=\sqrt{\frac{5+\sqrt{5}}{2}}, \quad t_{2}=\sqrt{\frac{5-\sqrt{5}}{2}} .
$$

The number of points of CR-II is

$$
n=4 m+5 \text {. }
$$

\subsection{Cubature rule of Type-III}

This part develops a more efficient cubature rule than CR-II. Denote

$$
\widehat{\boldsymbol{T}}_{1}=\left(\begin{array}{c}
t_{1} \\
-t_{1}
\end{array}\right), \quad \widehat{\boldsymbol{T}}_{2}=\left(\begin{array}{c}
t_{2} \\
t_{2}
\end{array}\right), \quad \widehat{\boldsymbol{O}}=\left(\begin{array}{l}
0 \\
0
\end{array}\right)
$$


The values of $t_{1}$ and $t_{2}$ are given in Eq.(19). Following Table 3 , a matrix $\boldsymbol{M}$ of size $(2 D+2) \times D$ can be constructed:

$$
M=\left(\begin{array}{cccccc}
\widehat{T}_{1} & \widehat{O} & \widehat{O} & \cdots & \widehat{O} & \widehat{O} \\
\widehat{T}_{2} & \widehat{T}_{1} & \widehat{O} & \cdots & \widehat{O} & \widehat{O} \\
\widehat{O} & \widehat{T}_{2} & \widehat{T}_{1} & \cdots & \widehat{O} & \widehat{O} \\
& \vdots & & & & \\
\widehat{O} & \widehat{O} & \widehat{O} & \cdots & \widehat{T}_{2} & \widehat{T}_{1} \\
\widehat{O} & \widehat{O} & \widehat{O} & \cdots & \widehat{O} & \widehat{T}_{2}
\end{array}\right)
$$

Let $\boldsymbol{I}_{(2 D+2) 2^{R} \times 1}$ be a column of size $(2 D+2) 2^{R} \times 1$, whose entries are 1 . Let $\boldsymbol{O}_{1 \times m}$ be a zero row of size $1 \times m$, the quadrature weights and points of CR-III are presented in Table 4 .

Table 4. The quadrature weights and point of cubature rule of Type-III.

\begin{tabular}{|l|l|}
\hline Weight & Point \\
\hline $1-w(2 D+2)$ & $\boldsymbol{O}_{1 \times m}$ \\
\hline$\frac{w}{2^{R}} \cdot \boldsymbol{I}_{(2 D+2) 2^{R} \times 1}$ & $\widehat{\boldsymbol{H}}_{2^{R} \times\left(2^{R}-1\right)} \otimes \boldsymbol{M}$ \\
\hline
\end{tabular}

In Table 4, “” denotes Kronecker product, $\widehat{\boldsymbol{H}}_{2^{R} \times\left(2^{R}-1\right)}$ is Hadamard matrix, which is given by the algorithm in Appendix B. The value of $R$ should satisfy

$$
D\left(2^{R}-1\right) \geq m
$$

In the case $D\left(2^{R}-1\right)>m$, the first $m$ columns of " $\widehat{\boldsymbol{H}}_{2^{R} \times\left(2^{R}-1\right)} \otimes \boldsymbol{M}$ " in Table 4 are considered, the number of points of CR-III is

$$
n=(2 D+2) 2^{R}+1
$$

\section{Computational procedures}

This section illustrates procedures of the proposed methods to calculate $E\left[Y^{k}\right]$ in Eq.(9):

1) Represent each POPF inputs by Hermite polynomial model in Eq.(1), determine the correlation matrix $\boldsymbol{R}_{\boldsymbol{Z}}$ by Eq. (2)

2) Determine the quadrature weights $p_{s}$ and points $\boldsymbol{t}_{s}(s=1, \ldots, n)$ by cubature rules in Section 3 .

3) Transform $\boldsymbol{t}_{s}(s=1, \ldots, n)$ to the original probability space by Eq.(5).

4) Perform deterministic OPF calculations at each quadrature point $\boldsymbol{x}_{s}(s=1, \ldots, n)$, calculate raw moments of POPF outputs by Eq.(9), obtain the mean $\mu_{y}$ and standard deviation $\sigma_{y}$ of each POPF outputs:

$$
\mu_{y}=E[Y], \quad \sigma_{y}=\sqrt{E\left[Y^{2}\right]-\mu_{y}^{2}}
$$




\section{Case study}

In this section, case studies are performed on IEEE 57-bus system ${ }^{1}$ and IEEE 118-bus system ${ }^{2}$.

\subsection{IEEE 57-bus system}

The IEEE 57-bus system includes 42 loads, 57 buses and 80 lines. The load demands are treated as POPF inputs, whose stochastic behavior is represented by Hermite polynomial model in Eq.(1):

$$
X_{i}=\Omega_{i}\left(Z_{i}\right)=\mu_{i}+\frac{\sigma_{i}\left[H_{1}\left(Z_{i}\right)+a_{i, 2} H_{2}\left(Z_{i}\right)+a_{i, 3} H_{3}\left(Z_{i}\right)\right]}{\sqrt{6 a_{i, 3}^{2}+2 a_{i, 2}^{2}+1}}, \quad(i=1, \ldots, 42),
$$

where $\mu_{i}$ equals the base case data, and $\sigma_{i}=2 \mu_{i} / 100$.

In order to check the linearity of the function relationship between POPF inputs and outputs, all POPF inputs are assumed to follow normal distributions. For $X_{i}$ represented by Eq.(24), it has:

$$
a_{i, 2}=a_{i, 3}=0, \quad(i=1, \ldots, 42)
$$

and the following scenarios are considered:

Case-I: the load demands are independent of each other.

Case-II the load demands are correlated with each other, and the correlation coefficient is 0.5 .

Because the sum of normal random variables is still normal, for Case-I and Case-II, if the POPF outputs in Eq.(7) can be expressed as a linear sum of $X_{i}(i=1, \ldots, 42)$ :

$$
Y=H(\boldsymbol{X})=a_{0}+\sum_{i=1}^{42} b_{i} X_{i}
$$

then $Y$ would also follow the normal distribution; the skewness of $Y$ must be 0 , and the kurtosis of $Y$ must be 3. If the skewness (kurtosis) of $Y$ shows an obvious deviation with respect to 0 (3), it implies that $Y$ is not normal, and the linear model in Eq.(25) does not provide an accurate representation for the function relationship between $Y$ and $\boldsymbol{X}$.

In this part, MCS with $10^{5}$ trials is employed to calculate the skewness and kurtosis of voltage $(V)$, phase angle $(\Theta)$, active power flow $(P l)$ and reactive power flow $(Q l)$, which are depicted in Fig. 1 and Fig. 2. An inspection of Fig. 1 and Fig. 2 indicates that, for most POPF outputs, the skewness is close to 0, the kurtosis is close to 3 , which means that they can be well characterized by near-normal distributions. However, the skewness of some angles shows an obvious deviation with respect to 0 , these POPF outputs cannot be related to POPF inputs by the linear model in Eq.(25).

Note that the POPF inputs of Case-II are correlated with each other, the interactive uncertainty of POPF inputs is more significant than that of Case-I. Table 5 presents the minimum and maximum values of skewness and kurtosis, the results indicate that the nonnormality of POPF outputs of Case-II is more severe than that

\footnotetext{
${ }^{1}$ IEEE 57-Bus System. [Online]. 2021. URL https://icseg.iti.illinois.edu/ieee-57-bus-system/

${ }^{2}$ IEEE 118-Bus System. [Online]. 2021. URL https://icseg.iti.illinois.edu/ieee-118-bus-system/
} 
Table 5. The minimum and maximum values of skewness and kurtosis of POPF outputs.

\begin{tabular}{|l|llll|lllll|}
\hline \multirow{2}{*}{} & \multicolumn{4}{|c|}{ Case-I } & \multicolumn{4}{c|}{ Case-II } \\
\cline { 2 - 10 } & \multicolumn{2}{|c}{ Skewness } & \multicolumn{2}{c|}{ Kurtosis } & \multicolumn{2}{c|}{ Skewness } & \multicolumn{3}{c|}{ Kurtosis } \\
\cline { 2 - 9 } & min. & max. & min. & max. & min. & max. & min. & max. \\
\hline$V$ & -0.047 & 0.091 & 2.703 & 3.097 & -0.412 & 0.229 & 2.936 & 3.318 \\
\hline$\Theta$ & -0.549 & 0.131 & 2.974 & 4.012 & -1.231 & 0.367 & 2.948 & 4.991 \\
\hline$P l$ & -0.262 & 0.283 & 2.925 & 3.404 & -0.603 & 1.021 & 2.930 & 5.172 \\
\hline$Q l$ & -0.171 & 0.175 & 2.933 & 3.597 & -0.469 & 0.544 & 2.943 & 4.759 \\
\hline
\end{tabular}

of Case-I, therefore, the interactive uncertainties among POPF inputs do have impacts on POPF outputs. In summary, the POPF outputs should be related to POPF inputs by the following model:

$$
\begin{gathered}
Y=H(\boldsymbol{X}) \simeq a_{0}+\sum_{i=1}^{m} b_{i} X_{i}+\sum_{i=1}^{m} c_{i} X_{i}^{2}+\sum_{1 \leq i<j \leq m} e_{i, j} X_{i} X_{j}, \\
Y^{2}=H^{2}(\boldsymbol{X}) \simeq\left(a_{0}+\sum_{i=1}^{m} b_{i} X_{i}+\sum_{i=1}^{m} c_{i} X_{i}^{2}+\sum_{1 \leq i<j \leq m} e_{i, j} X_{i} X_{j}\right)^{2} .
\end{gathered}
$$

According to Eq.(26), in order to accurately obtain the moments of POPF outputs, the weights and points in Eq.(9) should commit the following equations:

$$
\begin{array}{rlrl}
\sum_{s=1}^{n} p_{s} t_{s, i} & =0, & \sum_{s=1}^{n} p_{s} t_{s, i}^{3}=0 ; & \\
\sum_{s=1}^{n} p_{s} & =1, & \sum_{s=1}^{n} p_{s} t_{s, i}^{2}=1, & \sum_{s=1}^{n} p_{s} t_{s, i}^{4}=3 ; \\
\sum_{s=1}^{n} p_{s} t_{s, i} t_{s, j} & =0, & \sum_{s=1}^{n} p_{s} t_{s, i}^{2} t_{s, j}=0, & \sum_{s=1}^{n} p_{s} t_{s, i} t_{s, j}^{2}=0 ; \\
\sum_{s=1}^{n} p_{s} t_{s, i}^{2} t_{s, j}^{2} & =1 . &
\end{array}
$$

Table 6 shows the moment matching equations satisfied by cubature rules in Section 3 and Zhao's PEM in [23], the last row gives the number of quadrature points.

To comprehensively illustrate the accuracy of these algorithms, the skewness of each load demand is set to be 0.5 , the kurtosis is set to be 4 , it has:

$$
a_{i, 2}=0.072782, \quad a_{i, 3}=0.024588, \quad(i=1, \ldots, 42)
$$

and the following two cases are considered:

Case-III: the skewness of each load demand is 0.5 , the kurtosis of each load demand is 4 , the load demands are independent of each other. 
XIAO/Turk J Elec Eng \& Comp Sci

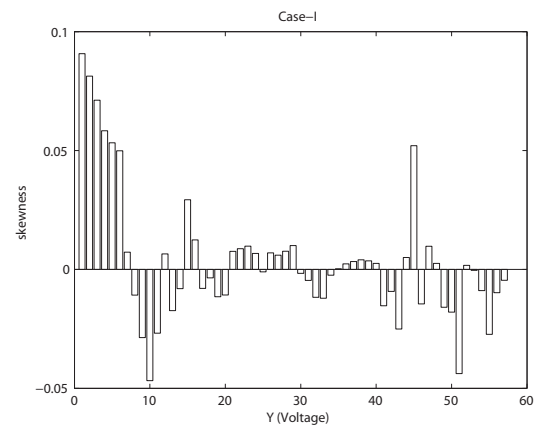

Skewness $(V)$

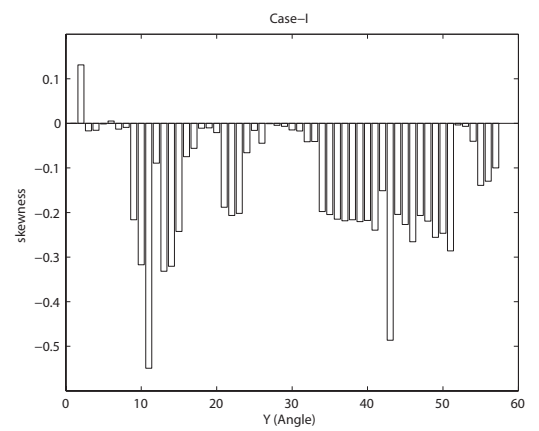

Skewness $(\Theta)$

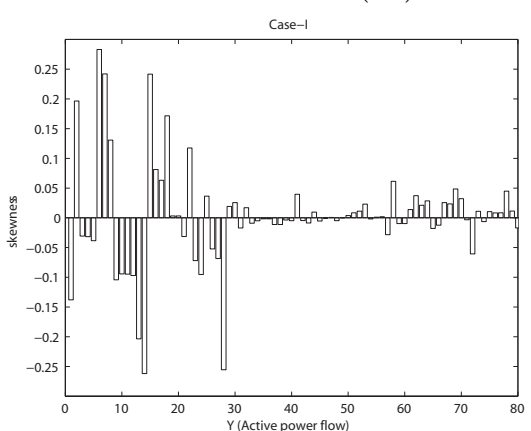

Skewness $(P l)$

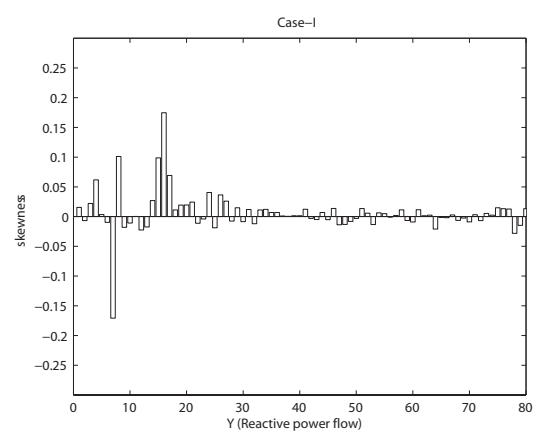

Skewness $(Q l)$

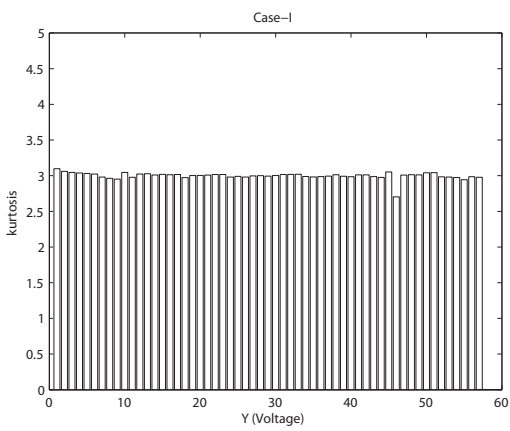

Kurtosis $(V)$

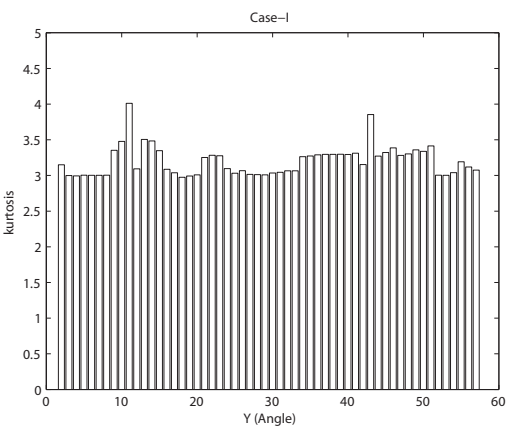

Kurtosis $(\Theta)$

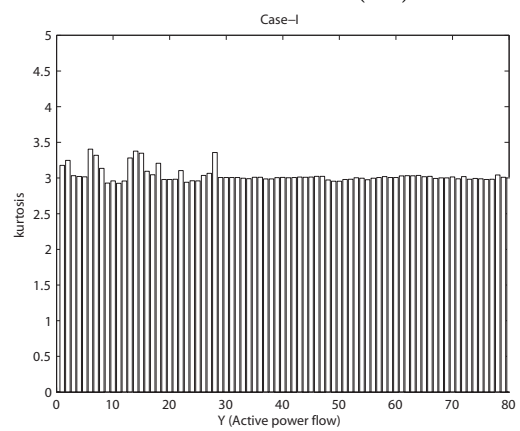

Kurtosis $(P l)$

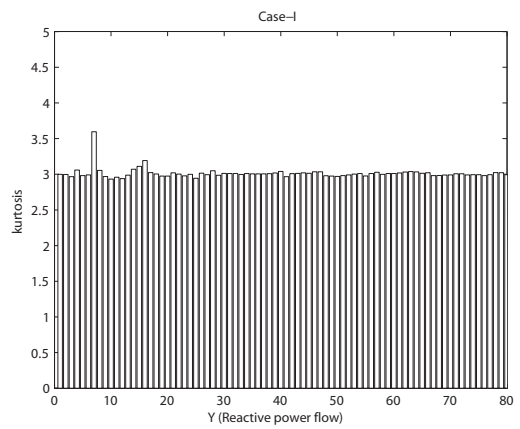

Kurtosis $(Q l)$

Figure 1. The skewness and kurtosis of POPF outputs (Case-I). 

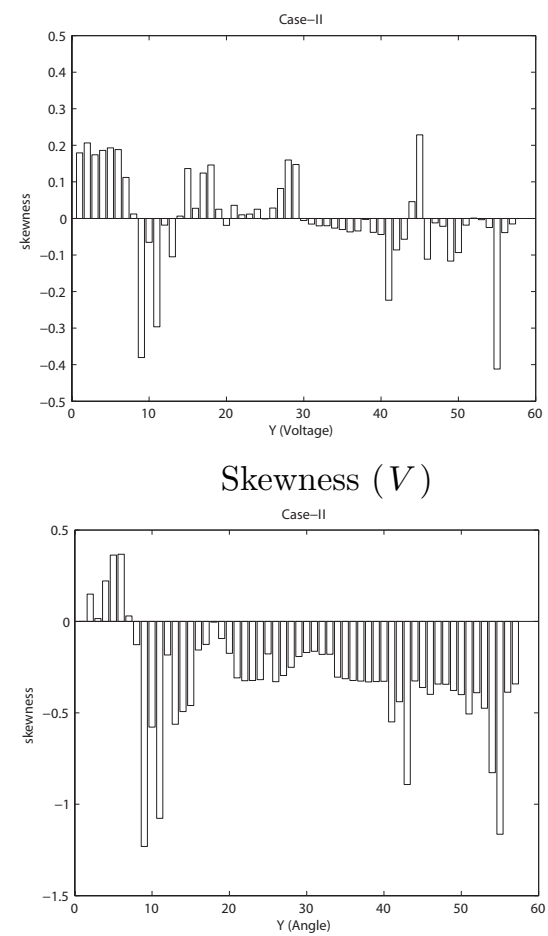

Skewness $(\Theta)$

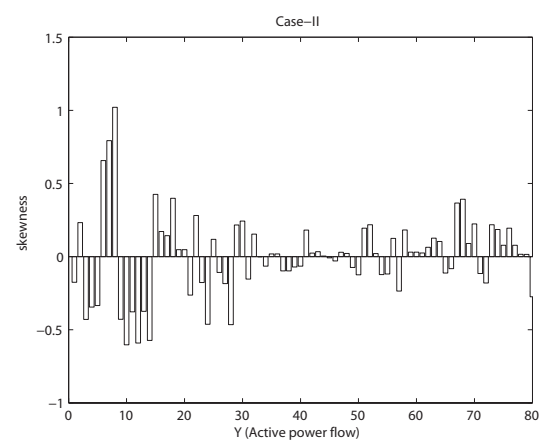

Skewness $(P l)$

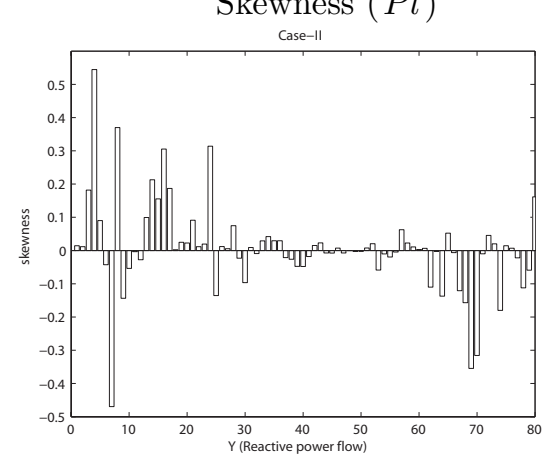

Skewness $(Q l)$

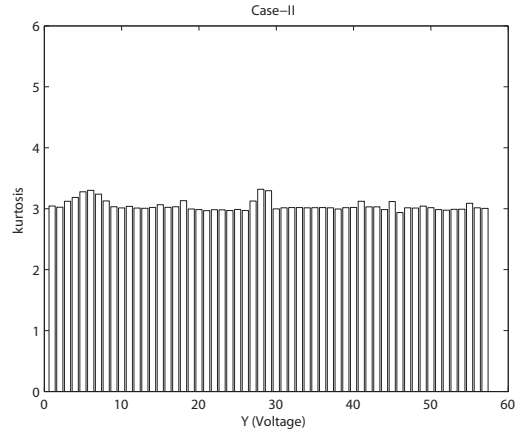

Kurtosis $(V)$

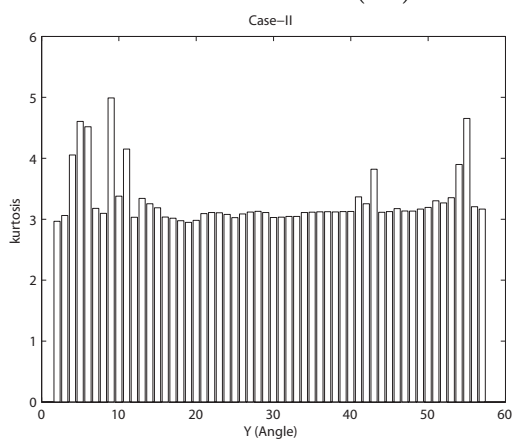

Kurtosis $(\Theta)$

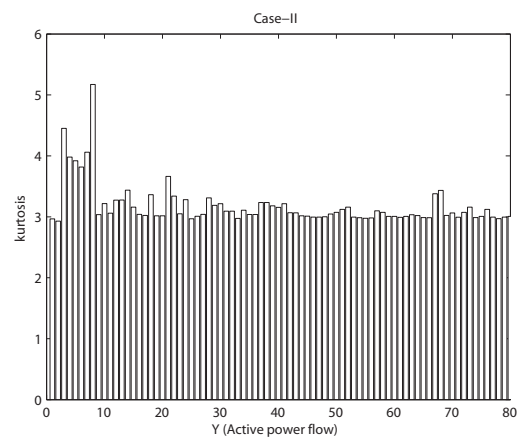

Kurtosis $(P l)$

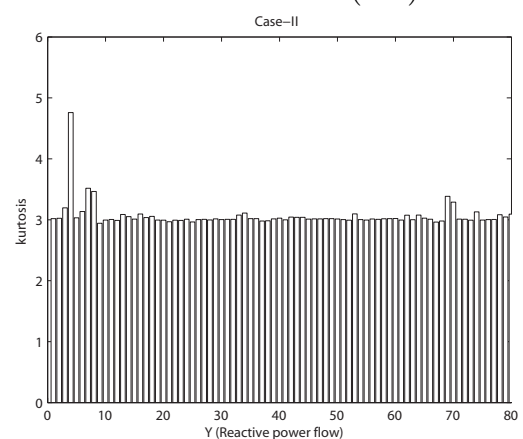

Kurtosis $(Q l)$

Figure 2. The skewness and kurtosis of POPF outputs (Case-II). 
Table 6. Checking cubature rules and PEM by moment matching equations.

\begin{tabular}{|l|l|l|l|l|}
\hline & CR-I & CR-II & CR-III & PEM $(2 m+1)$ \\
\hline Eq.(27) & all & all & all & all \\
\hline Eq.(28) & all & all & all & all \\
\hline Eq.(29) & all & all & most & all \\
\hline Eq.(30) & all & some & most & none \\
\hline$n$ & $2 m^{2}+1$ & $4 m+5$ & $(2 D+2) 2^{R}+1$ & $2 m+1$ \\
\hline
\end{tabular}

Case-IV: the skewness of each load demand is 0.5 , the kurtosis of each load demand is 4 , the load demands are correlated with each other, and the correlation coefficient is 0.5 , the equivalent correlation coefficient in normal space is 0.491 (see Eq.(2)).

The following error index is defined to assess the performance of algorithms in Table 6 :

$$
\bar{\varepsilon}_{k}=\frac{1}{N} \sum_{r=1}^{N}\left|\frac{\mu_{k, r}^{M C S}-\mu_{k, r}^{*}}{\mu_{k, r}^{M C S}}\right| \times 100[\%]
$$

where $\mu_{k, r}^{M C S}$ is the mean $(k=1)$ or standard deviation $(k=2)$ of $r$ th POPF outputs from MCS, $\mu_{k, r}^{*}$ is the one estimated by algorithms in Table $6 . \bar{\varepsilon}_{k}$ is the average absolute relative error. $N$ is the number of the output variables in the system.

The parameters of CR-III are:

$$
D=6, \quad R=3 .
$$

With the benchmark from MCS with $10^{5}$ trials, the results are summarized in Table $7(V, \Theta, P l$, and $Q l$ denote the voltage, phase angle, active power flow, and reactive power flow respectively). For the four algorithms in Table 7, if an algorithm can meet more moment matching equations, it is expected to yield more accurate results. Along with Table 6, the following conclusions can be drawn:

1) An inspection of Eq.(26) and Eq.(23) indicates that, in comparison to $E[Y], E\left[Y^{2}\right]$ is related to POPF inputs by a function with a higher nonlinearity; therefore, values of $\bar{\varepsilon}_{2}$ are generally larger than those of $\bar{\varepsilon}_{1}$.

2) According to Table 6, the major difference among the implemented four algorithms lies in how well they can commit Eq.(30). Compared with the proposed cubature rules, PEM can satisfy no equations in Eq.(30), and thus performs poorly for calculating standard deviations in Case-II and Case-IV. Because CR-I and CR-III can meet more equations in Eq.(30) than CR-II, these two cubature rules perform better than CR-II.

3) For Case-III and Case-IV, the POPF inputs are nonnormal, $\Omega_{i}(\cdot)$ in Eq.(24) is a third-order polynomial. According to Eq.(8), the function relationship between $Y$ and $\boldsymbol{U}$ is more severely nonlinear than that of Case-I and Case-II, where $\Omega_{i}(\cdot)$ is linear. When calculating mean and standard deviations for Case-III and Case-IV, the cubature rule should satisfy more equations in Eqs.(11)-(14). While by checking the weights and points, CR-I cannot well match equations in Eq.(12) with $r_{i} \geq 3$ and equations in Eq.(14) 
Table 7. Error in \% of cubature rules and PEM (IEEE 57-bus system).

\begin{tabular}{|c|c|c|c|c|c|c|c|c|c|c|c|}
\hline \multicolumn{6}{|c|}{ Independent POPF inputs } & \multicolumn{6}{|c|}{ Correlated POPF inputs } \\
\hline \multicolumn{2}{|c|}{ Case-I } & CR-I & CR-II & CR-III & PEM & \multicolumn{2}{|c|}{ Case-II } & CR-I & CR-II & CR-III & PEM \\
\hline \multirow{2}{*}{$V$} & $\bar{\varepsilon}_{1}$ & 0.0002 & 0.0195 & 0.0002 & 0.0006 & \multirow{2}{*}{$V$} & $\bar{\varepsilon}_{1}$ & 0.0008 & 0.0196 & 0.0004 & 0.0196 \\
\hline & $\bar{\varepsilon}_{2}$ & 0.90 & 0.75 & 0.78 & 0.41 & & $\bar{\varepsilon}_{2}$ & 2.08 & 1.93 & 2.01 & 2.44 \\
\hline \multirow{2}{*}{$\Theta$} & $\bar{\varepsilon}_{1}$ & 0.10 & 0.31 & 0.02 & 0.23 & \multirow{2}{*}{$\Theta$} & $\bar{\varepsilon}_{1}$ & 0.30 & 0.55 & 0.09 & 2.09 \\
\hline & $\bar{\varepsilon}_{2}$ & 1.13 & 1.26 & 1.07 & 1.28 & & $\bar{\varepsilon}_{2}$ & 1.99 & 2.31 & 1.85 & 5.49 \\
\hline \multirow{2}{*}{$P l$} & $\bar{\varepsilon}_{1}$ & 0.03 & 0.08 & 0.03 & 0.08 & \multirow{2}{*}{$P l$} & $\bar{\varepsilon}_{1}$ & 0.05 & 0.74 & 0.03 & 1.72 \\
\hline & $\bar{\varepsilon}_{2}$ & 1.03 & 1.16 & 0.76 & 1.07 & & $\bar{\varepsilon}_{2}$ & 1.33 & 1.95 & 2.02 & 6.12 \\
\hline \multirow{2}{*}{$Q l$} & $\bar{\varepsilon}_{1}$ & 0.03 & 0.09 & 0.01 & 0.06 & \multirow{2}{*}{$Q l$} & $\bar{\varepsilon}_{1}$ & 0.07 & 0.75 & 0.04 & 0.77 \\
\hline & $\bar{\varepsilon}_{2}$ & 0.72 & 0.67 & 0.64 & 0.37 & & $\bar{\varepsilon}_{2}$ & 1.62 & 1.70 & 1.38 & 2.17 \\
\hline \multicolumn{2}{|l|}{$n$} & 3529 & 173 & 113 & 83 & \multicolumn{2}{|c|}{$n$} & 3529 & 173 & 113 & 83 \\
\hline \multicolumn{2}{|c|}{ Time (s) } & 1245.4 & 61.1 & 39.9 & 29.3 & \multicolumn{2}{|c|}{ Time (s) } & 1245.4 & 61.1 & 39.9 & 29.3 \\
\hline \multicolumn{2}{|c|}{ Case-III } & CR-I & CR-II & CR-III & PEM & \multicolumn{2}{|c|}{ Case-IV } & CR-I & CR-II & CR-III & PEM \\
\hline \multirow{2}{*}{$V$} & $\bar{\varepsilon}_{1}$ & 0.0001 & 0.0003 & 0.0001 & 0.0001 & \multirow{2}{*}{$V$} & $\bar{\varepsilon}_{1}$ & 0.0016 & 0.0003 & 0.0003 & 0.0008 \\
\hline & $\bar{\varepsilon}_{2}$ & 2.13 & 0.46 & 0.55 & 1.39 & & $\bar{\varepsilon}_{2}$ & 2.61 & 2.06 & 1.87 & 7.05 \\
\hline \multirow{2}{*}{$\Theta$} & $\bar{\varepsilon}_{1}$ & 0.11 & 0.04 & 0.03 & 0.06 & \multirow[t]{2}{*}{$\Theta$} & $\bar{\varepsilon}_{1}$ & 0.44 & 0.13 & 0.06 & 0.33 \\
\hline & $\bar{\varepsilon}_{2}$ & 2.27 & 1.72 & 1.33 & 2.42 & & $\bar{\varepsilon}_{2}$ & 1.90 & 2.26 & 2.08 & 8.57 \\
\hline \multirow{2}{*}{$P l$} & $\bar{\varepsilon}_{1}$ & 0.18 & 0.02 & 0.01 & 0.02 & \multirow{2}{*}{$P l$} & $\bar{\varepsilon}_{1}$ & 0.41 & 0.05 & 0.03 & 0.14 \\
\hline & $\bar{\varepsilon}_{2}$ & 2.05 & 0.96 & 0.78 & 1.33 & & $\bar{\varepsilon}_{2}$ & 2.24 & 2.64 & 1.42 & 9.35 \\
\hline \multirow{2}{*}{$Q l$} & $\bar{\varepsilon}_{1}$ & 0.05 & 0.03 & 0.01 & 0.02 & \multirow{2}{*}{$Q l$} & $\bar{\varepsilon}_{1}$ & 0.54 & 0.02 & 0.03 & 0.08 \\
\hline & $\bar{\varepsilon}_{2}$ & 1.94 & 0.86 & 0.53 & 1.10 & & $\bar{\varepsilon}_{2}$ & 2.48 & 2.43 & 1.58 & 9.29 \\
\hline \multicolumn{2}{|l|}{$n$} & 3529 & 173 & 113 & 83 & \multicolumn{2}{|l|}{$n$} & 3529 & 173 & 113 & 83 \\
\hline \multicolumn{2}{|c|}{ Time (s) } & 1245.4 & 61.1 & 39.9 & 29.3 & \multicolumn{2}{|c|}{ Time (s) } & 1245.4 & 61.1 & 39.9 & 29.3 \\
\hline
\end{tabular}

with $\left(r_{1}+\cdots+r_{m}\right) \geq 3$ :

$$
\begin{array}{lc}
\sum_{s=1}^{n} p_{s} t_{s, i}^{2 r_{i}}<0<E\left[U_{i}^{2 r_{i}}\right], & \left(r_{i} \geq 3\right) \\
\sum_{s=1}^{n} p_{s} t_{s, 1}^{2 r_{1}} \cdots t_{s, i}^{2 r_{i}} \cdots t_{s, m}^{2 r_{m}} \gg \prod_{i=1}^{m} E\left[U_{i}^{2 r_{i}}\right], & \left(r_{1}+\cdots+r_{m}\right) \geq 3 .
\end{array}
$$

As shown in Table 6, CR-I demonstrates a lower accuracy than CR-III in Case-III and Case-IV.

4) For the POPF problem with correlated inputs, the interactive uncertainty among POPF inputs would be more significant than that of the independent case. An inspection of Table 7 indicates that all four algorithms show a higher accuracy in Case-I and Case-III, where POPF inputs are independent of each other.

\subsection{IEEE 118-bus system}

In this part, a case study is performed on a modified IEEE 118-bus system, of which the detailed description can be found in [6]. The POPF inputs comprise load demands at 99 nodes and wind speeds at 14 wind farms. 
The wind speed is represented by Weibull distribution with scale parameter 10.7 and shape parameter 3.97, the parameters of corresponding Hermite polynomial model are [19]:

$$
\mu=9.694361, \quad \sigma=2.738277, \quad a_{2}=-0.012739, \quad a_{3}=0.002615 .
$$

For the tested IEEE 118-bus system, the number of POPF inputs is 113, and the parameters of CR-III are

$$
D=8, \quad R=4
$$

With benchmark from MCS with $10^{6}$ trials, values of $\bar{\varepsilon}_{1}$ and $\bar{\varepsilon}_{2}$ are calculated by Eq.(32), which are shown in Table 8. As shown in Table 6 and Table 8, CR-III can meet most equations in Eq.(30), and yields results with satisfactory accuracy; CR-II can commit some equations in Eq.(30), and gives good estimations for mean values of POPF outputs; the poor performance of PEM demonstrates the importance of satisfying equations in Eq.(30), which represents the interactive uncertainty effects of POPF inputs on outputs.

Table 8. Error in \% of cubature rules and PEM (IEEE 118-bus system).

\begin{tabular}{|l|l|l|l|l|l|}
\hline \multirow{2}{*}{$V$} & & CR-I & CR-II & CR-III & PEM \\
\cline { 2 - 6 } & $\bar{\varepsilon}_{1}$ & 0.010 & 0.007 & 0.003 & 0.008 \\
\cline { 2 - 6 }$\Theta$ & $\bar{\varepsilon}_{2}$ & 3.60 & 5.90 & 2.08 & 9.40 \\
\hline \multirow{2}{*}{$\Theta$} & $\bar{\varepsilon}_{1}$ & 1.36 & 0.84 & 0.43 & 1.37 \\
\cline { 2 - 6 } & $\bar{\varepsilon}_{2}$ & 2.79 & 2.66 & 2.23 & 3.14 \\
\hline \multirow{2}{*}{$P l$} & $\bar{\varepsilon}_{1}$ & 1.47 & 0.94 & 0.67 & 4.44 \\
\cline { 2 - 6 } & $\bar{\varepsilon}_{2}$ & 4.32 & 4.08 & 1.96 & 5.08 \\
\hline \multirow{2}{*}{$Q l$} & $\bar{\varepsilon}_{1}$ & 1.04 & 0.75 & 0.87 & 2.25 \\
\cline { 2 - 6 } & $\bar{\varepsilon}_{2}$ & 4.52 & 4.16 & 2.19 & 5.00 \\
\hline \multirow{2}{*}{$n$} & & 25539 & 457 & 289 & 227 \\
\hline \multicolumn{2}{|c|}{$T$ Time (s) } & 29009.8 & 519.1 & 328.3 & 257.8 \\
\hline
\end{tabular}

If CR-III is checked by using equations in Eq.(12) with $r_{i} \geq 3$ and equations in Eq.(14) with $\left(r_{1}+\cdots+\right.$ $\left.r_{m}\right) \geq 3$, it has:

$$
\begin{array}{ll}
0<\sum_{s=1}^{n} p_{s} t_{s, i}^{2 r_{i}}<E\left[U_{i}^{2 r_{i}}\right], & \left(r_{i} \geq 3\right) \\
0<\sum_{s=1}^{n} p_{s} t_{s, 1}^{2 r_{1}} \cdots t_{s, i}^{2 r_{i}} \cdots t_{s, m}^{2 r_{m}}<\prod_{i=1}^{m} E\left[U_{i}^{2 r_{i}}\right], & \left(r_{1}+\cdots+r_{m}\right) \geq 3 .
\end{array}
$$

A comparison between Eq.(33) and Eq.(34) illustrates the lower accuracy of CR-I with respect to CR-III.

\section{Conclusion}

This paper is devoted to revealing some properties of POPF problem. In conjunction with Hermite polynomial model, Nataf transformation is introduced to simulate different operating conditions of power systems. In the context of moment matching equations, three cubature rules are developed for POPF computation. Through numerical experiments and theoretical analysis, the following conclusions can be drawn: 
1) The polynomial model in Eq.(26) can well approximate the function relationship between POPF input and outputs.

2) The interactive uncertainty among POPF inputs has a significant impact on POPF output, the employed cubature rule should well satisfy moment matching equations in Eq.(13) and Eq.(14).

3) If POPF inputs follow normal distributions, cubature rules derived from Eqs.(27)-(30) would yield accurate estimates for means and standard deviations of POPF outputs; if POPF inputs are nonnormal random variables, the cubature rule should satisfy more equations in Eqs.(11)-(14).

4) Even if the cubature rule cannot exactly match Eqs.(11)-(14), the sum of weights and points by moment matching equations should satisfy Eq.(34) rather than Eq.(33).

\section{References}

[1] Schellenberg, A., Rosehart, W., Aguado, J.. Introduction to cumulant-based probabilistic optimal power flow (P-OPF). IEEE Transactions on Power Systems 2005;20(2):1184-1186.

[2] Rahmani, S., Amjady, N.. A new optimal power flow approach for wind energy integrated power systems. Energy 2017;134:349-359.

[3] Cao, J., Yan, Z.. Probabilistic optimal power flow considering dependences of wind speed among wind farms by pair-copula method. International Journal of Electrical Power \& Energy Systems 2017;84:296-307.

[4] Li, G., Lu, W., Bian, J., Qin, F., Wu, J.. Probabilistic optimal power flow calculation method based on adaptive diffusion kernel density estimation. Frontiers in Energy Research 2019;7(128):1-10.

[5] Lin, W., Yang, Z., Yu, J., Bao, S., Dai, W.. Toward fast calculation of probabilistic optimal power flow. IEEE Transactions on Power Systems 2019;34(4):3286-3288.

[6] Zou, B., Xiao, Q.. Solving probabilistic optimal power flow problem using quasi Monte Carlo method and ninth-order polynomial normal transformation. IEEE Transactions on Power Systems 2014;29(1):300-306.

[7] Xie, Z., Ji, T., Li, M., Wu, Q.. Quasi-Monte Carlo based probabilistic optimal power flow considering the correlation of wind speeds using copula function. IEEE Transactions on Power Systems 2018;33(2):2239-2247.

[8] Xiao, Q., Zhou, S., Xiao, H.. Probabilistic optimal power flow analysis incorporating correlated wind sources. International Transactions on Electrical Energy Systems 2020;e12441:1-24.

[9] Sun, W., Zamani, M., Zhang, H.T., Li, Y.. Probabilistic optimal power flow with correlated wind power uncertainty via Markov chain quasi-Monte-Carlo sampling. IEEE Transactions on Industrial Informatics 2019;15(11):6058-6069.

[10] Sun, W., Zamani, M., Hesamzadeh, M.R., Zhang, H.T.. Data-driven probabilistic optimal power flow with nonparametric Bayesian modeling and inference. IEEE Transactions on Smart Grid 2020;11(2):1077-1090.

[11] Sun, X., Tu, Q., Chen, J., Zhang, C., Duan, X.. Probabilistic load flow calculation based on sparse polynomial chaos expansion. IET Generation, Transmission \& Distribution 2018;12(11):2735-2744.

[12] Yin, H., Zivanovic, R.. Probabilistic power flow computation using collocation method and including correlation modeling. International Transactions on Electrical Energy Systems 2019;29(4):2796-2796.

[13] Alavi, S.A., Ahmadian, A., Aliakbar-Golkar, M.. Optimal probabilistic energy management in a typical micro-grid based-on robust optimization and point estimate method. Energy Conversion and Management 2015;95:314-325.

[14] Shargh, S., Mohammadi-Ivatloo, B., Seyedi, H., Abapour, M., et al. Probabilistic multi-objective optimal power flow considering correlated wind power and load uncertainties. Renewable Energy 2016;94:10-21. 
[15] Xiao, Q.. Comparing three methods for solving probabilistic optimal power flow. Electric Power Systems Research 2015;124:92-99.

[16] Tamtum, A., Schellenberg, A., Rosehart, W.D.. Enhancements to the cumulant method for probabilistic optimal power flow studies. IEEE Transactions on Power Systems 2009;24(4):1739-1746.

[17] Aien, M., Fotuhi-Firuzabad, M., Aminifar, F.. Unscented transformation-based probabilistic optimal power flow for modeling the effect of wind power generation. Turkish Journal of Electrical Engineering \& Computer Sciences 2013;21(5):1284-1301.

[18] Xiao, Q., Zhou, S.. Comparing unscented transformation and point estimate method for probabilistic power flow computation. COMPEL-The International Journal for Computation and Mathematics in Electrical and Electronic Engineering 2018;37(3):1290-1303.

[19] Yang, L., Gurley, K.R., Prevatt, D.O.. Probabilistic modeling of wind pressure on low-rise buildings. Journal of Wind Engineering and Industrial Aerodynamics 2013;114:18-26.

[20] Xiao, Q., Zhou, S.. Matching a correlation coefficient by a Gaussian copula. Communications in Statistics-Theory and Methods 2019;48(7):1728-1747.

[21] Lebrun, R., Dutfoy, A.. An innovating analysis of the Nataf transformation from the copula viewpoint. Probabilistic Engineering Mechanics 2009;24(3):312-320.

[22] Xiao, Q., Zhou, S.. Probabilistic power flow computation using quadrature rules based on discrete Fourier transformation matrix. International Journal of Electrical Power \& Energy Systems 2019;104:472-480.

[23] Zhao, Y.G., Ono, T.. New point estimates for probability moments. Journal of Engineering Mechanics $2000 ; 126(4): 433-436$. 


\section{S1. Appendix A}

Let $Y$ denote a POPF output, let $U$ be a POPF input, which is assumed to follow the standard normal distribution, and

$$
Y=G(U) .
$$

According to the surrogate method, $G(\cdot)$ can be approximated by a weighted sum of Hermite polynomial:

$$
Y=H(U)=\sum_{k=0}^{\infty} a_{k} H_{k}(U) \simeq \sum_{k=0}^{m} a_{k} H_{k}(U)
$$

where $a_{k}(k=0,1, \ldots, m)$ are coefficients, $H_{k}(U)$ is the $k$ th-order Hermite polynomial, and

$$
H_{0}(U)=1, \quad H_{1}(U)=U, \quad H_{k}(U)=U H_{k-1}(U)-(k-1) H_{k-2}(U) .
$$

The Hermite polynomials have the following property:

$$
E\left[H_{0}(U)\right]=1, \quad E\left[H_{k}(U)\right]=0, \quad(k=1,2,3, \ldots) .
$$

According to Eq.(36), the mean of $Y$ is calculated by:

$$
E[Y] \simeq \sum_{k=0}^{m} a_{k} E\left[H_{k}(U)\right]=a_{0}
$$

The coefficients in Eq.(36) can be calculated by an $n$-point Gauss-Hermite quadrature rule $(n>m)$ :

$$
a_{k}=\frac{1}{k !} \sum_{s=1}^{n} p_{s} H_{k}\left(t_{s}\right) G\left(t_{s}\right), \quad(k=0,1, \ldots, m)
$$

where $p_{s}$ and $t_{s}(s=1, \ldots, n)$ are the quadrature weights and points, respectively.

Substitute Eq.(39) into Eq.(38), it has:

$$
E[Y] \simeq \sum_{k=0}^{m} a_{k} E\left[H_{k}(U)\right]=a_{0}=\frac{1}{0 !} \sum_{s=1}^{n} p_{s} H_{0}\left(t_{s}\right) G\left(t_{s}\right)=\sum_{s=1}^{n} p_{s} G\left(t_{s}\right) .
$$

As shown in Eq.(40), $E[Y]$ is actually calculated by the univariate Gauss-Hermite quadrature, which is also referred to as PEM.

\section{S2. Appendix B}

Here, an easy-to-use algorithm is presented to construct Hadamard matrix.

$$
\begin{aligned}
& \boldsymbol{M}^{(1)}=\left(\begin{array}{ll}
0 & 1 \\
1 & 0
\end{array}\right), \\
& \boldsymbol{M}^{(R)}=\left(\begin{array}{ll}
\boldsymbol{M}^{(R-1)} & 2^{R-1} \boldsymbol{E}_{2^{R-1} \times 2^{R-1}} \\
2^{R-1} \boldsymbol{E}_{2^{R-1} \times 2^{R-1}} & \boldsymbol{M}^{(R-1)}
\end{array}\right)
\end{aligned}
$$

where $\boldsymbol{E}_{2^{R-1} \times 2^{R-1}}$ is the identity matrix of size $2^{R-1} \times 2^{R-1}$. Denote $\lambda_{k}$ as the eigenvalue of $\boldsymbol{M}^{(R)}$, it has:

$$
\lambda_{k}=2 k-2^{R}-1, \quad\left(k=1, \ldots, 2^{R}\right) .
$$


XIAO/Turk J Elec Eng \& Comp Sci

Denote $\boldsymbol{\Lambda}=\operatorname{diag}\left(\lambda_{1}, \ldots, \lambda_{k}, \ldots, \lambda_{2}\right)$, denote $\boldsymbol{V}^{(R)}$ as the eigenvectors of $\boldsymbol{M}^{(R)}$, it has:

$$
\boldsymbol{M}^{(R)} \cdot \boldsymbol{\Lambda}=\boldsymbol{V}^{(R)} \cdot \boldsymbol{\Lambda}
$$

then, $\boldsymbol{V}^{(R)}$ would be the Hadamard matrix of order $2^{R}$. 